\title{
Evaluation of Dynamic Sensitivity Control Algorithm for IEEE 802.11ax
}

\author{
M. Shahwaiz Afaqui*, Eduard Garcia-Villegas*, Elena Lopez-Aguilera*, Graham Smith ${ }^{\dagger}$, Daniel Camps ${ }^{\ddagger}$ \\ *UPC-BarcelonaTech \\ ${ }^{\dagger}$ DSP Group \\ $\ddagger_{\text {i2CAT Foundation }}$
}

\begin{abstract}
The explosive growth in the usage of IEEE 802.11 network has resulted in dense deployments in diverse environments. Most recently, the IEEE working group has triggered the IEEE 802.11ax project, which aims to amend the current IEEE 802.11 standard to improve efficiency of dense WLANs. In this paper, we evaluate the Dynamic Sensitivity Control (DSC) Algorithm proposed for IEEE 802.11ax. This algorithm dynamically adjusts the Carrier Sense Threshold (CST) based on the average received signal strength. We show that the aggregate throughput of a dense network utilizing DSC is considerably improved (i.e. up to $20 \%$ ) when compared with the IEEE 802.11 legacy network.
\end{abstract}

\section{INTRODUCTION}

In recent years, growth in IEEE 802.11 Wireless Local Area Network (WLAN) technology has drastically increased due to its ease of deployment, convenience and cost efficiency. More and more WLAN devices are being deployed in different environments which encompass many Access Points (AP) and client stations working in geographically limited vicinity (e.g. residential buildings, etc.). At one end, this massive deployment indicates the need for more capacity. But on the other end, these environments generate multiple Overlapping Basic Service Set (OBSS) where interference from the neighboring devices degrades performance and throughput due to contention.

The need for improved performance and efficient methods to share the limited resources has resulted in extensive research being done on spatial reuse, interference and efficient resource sharing. The IEEE Standardization committee has actively continued to release new protocols that provide higher throughput, better modulation techniques, multi-streaming, and many other new features. Most recently, the IEEE-SA standards board approved IEEE 802.11ax that is expected to contemplate standardization of radio resource mechanisms to improve performance of IEEE 802.11 under dense deployments. This paper is intended to evaluate the carrier sensing amendments suggested by the IEEE 802.11ax to improve the efficiency of Wi-Fi networks in dense scenarios.

In IEEE 802.11 networks, carrier sensing methods are used to manage the medium access by different nodes communicating within the network. The IEEE standard defines two mode of operation for carrier sensing, Point Coordination Function (PCF) and Distributed Coordination Function (DCF) [1]. PCF was optionally proposed and due to its complexity, there are very few implementations. DCF is the dominant protocol used due its simple and distributed implementation. It is a contention based protocol that utilizes Carrier Sense Multiple Access with Collision Avoidance (CSMA/CA). CSMA/CA enforces all the stations to contend to gain access to the shared medium and provides each station equal probability to access the channel. Before initiating transmission, each node senses the medium, if the energy level measured exceeds a predefine threshold, the channel is sensed busy and the node defers communication. This threshold is called Carrier Sensing Threshold (CST). As discussed in the following, an optimal tuning of this parameter can improve efficiency, especially in dense scenarios.

\section{A. Related work}

Considerable amount of work has been done to study how physical carrier sensing mechanism affects the capacity and throughput of IEEE 802.11 based WLANs [2],[3]. The motivation behind this work is to improve the efficiency of those networks so that requirements for increased capacity and higher performance could be fulfilled. Authors in [4] demonstrate simple modifications that can be made in carrier sensing mechanism to increase the overall throughput in dense networks. They propose changes to be made in DCF over IEEE 802.11 networks that can result in added complexity due to additional signaling over the network. Nevertheless, their proposed scheme can be viewed as a step towards the design of an algorithm that dynamically changes CST of a node based on received power. Similarly in [5], the authors propose cognitive protocol for enabling and disabling virtual NAV and PCS. Their methods require additional information to be added to RTS/CTS control frames and they use a heuristic method to modify the CST.

The increase in performance achieved by optimally adjusting CST is revealed in [6], where the authors propose that for maximum throughput, the CST is linearly dependent on the nodal density. However, the authors have not mentioned the adjustment method and the throughputs are evaluated for regular topologies by adjusting different threshold values. In [7], the authors propose a localized spatio-temporal algorithm that jointly controls contention window and carrier sensing threshold to enhance the spatial reuse and optimize the overall throughput in the network.

As discussed in Section II, a side effect of tuning CST is the variation in the number of exposed and hidden nodes. Hidden and exposed node problem is investigated by [8], where the authors demonstrate that the throughput in network can be increased by tuning CST after every change in network topology. By doing so, the numbers of deferred transmissions are reduced. But the authors have not investigated their scheme in dense infrastructural network.

Recently, the IEEE 802.11ax task group ${ }^{1}$ [9] was estab-

${ }^{1}$ IEEE 802.11ax task group has evolved from HEW study group. 
lished to characterize modifications/amendments to be made in IEEE 802.11 physical (PHY) and Medium Access Control (MAC) layers to facilitate mode of operation that would be capable to improve average throughput per station in dense deployments. These amendments are expected to permit backward compatibility and coexistence with legacy IEEE 802.11 devices operating in the same bands. Residential, enterprise, indoor and outdoor hotspots are considered to be the main usage models of these amendments because, due to their user density, they are expected to suffer bottlenecks.

As mentioned in IEEE 802.11ax working documents [10], one of the main objectives is to improve efficiency in scenarios having high density of AP and non-AP stations by considerably increasing the spectral frequency reuse and interference management in OBSS. In this paper, we investigate the performance of DSC mechanism proposed in [11] to increase the area throughput in dense apartment block. In DSC method, CST is changed according to channel conditions, where each station measures the power of beacons received from the AP and varies its CST according to the received power. We use NS-3 [12] simulator to measure the performance of a dense WLAN network that contains multiple OBSS and employ the DSC algorithm. We compare scenarios where IEEE 802.11g and IEEE 802.11n dense networks use and do not use DSC to increase throughput.

The remainder of the paper is organized as follows. In Section 2, we present the problem of carrier sensing is dense building environments. In Section 3, the concept and implementation details of Dynamic Sensitivity Control Algorithm are exposed. The details of simulation environment are given in section 4. In section 5, the performance evaluation of DSC in dense building environment is presented.

\section{PROBLEMS ASSOCIATED WITH CARRIER SENSING IN DENSE NETWORK}

As explained in section I, IEEE 802.11 utilizes CSMA/CA where the stations are made to listen before transmitting over the shared channel. PCS method is responsible for reporting status of the medium to the MAC layer and leverages the Clear Channel Assessment (CCA) modules implemented at the physical layer. The CCA module is able to sense the channel (i.e. busy or idle) by measuring the received energy.

Despite of improvements in network conditions caused by the usage of DCF, it has inherent flaws, such as the hidden and exposed node problems. Hidden node problem is caused when a node that is located outside the sensing range of the transmitter is able to interfere in the ongoing transmission from the transmitter to the receiver. Since the hidden node is not silenced, it causes collisions at the receiver. On the contrary, exposed node problem occurs when a node is needlessly silenced to concurrently transmit, even though the node is not able to generate ample interference that could cause collisions at the receiver.

Both hidden and exposed nodes result in a decreased overall throughput. As explained in [13], the exposed node problem creates severe scalability problems; the total throughput of IEEE 802.11 network reaches a limit when the density of APs is increased.

However, a phenomenon called Capture effect [14] illustrates that, in case of collision, the receivers can successfully decode frames having stronger Signal to Noise
Ratio (SNR). Capture effect, along with the standardized four-way RTS/CTS transmission mode, can alleviate the effect of hidden nodes (to some extent) over the cost of fairness.

Locally on each node, the CST parameter used by each node has a direct impact on perceived condition of the channel. A lower CST used by the node results in reduced concurrent transmissions whereas a high CST increases interference amongst the concurrent transmissions. Thus, the value of CST used by each node affects the presence and number of hidden and exposed nodes. When a node decreases its CST, it can sense nodes present further away and can reduce number of hidden nodes affecting its transmission. But the node can also gratuitously sense transmissions intended for other receivers not in range, and thus can increase exposed nodes. As a result, the CST for a node can be tuned so as to maximize throughput and balance the impact of hidden and exposed nodes.

Given a transmitter $i$, the CCA returns channel idle indication at time $t$ to the MAC layer if

$$
\sum_{k \in i n t} P_{r}\left(d_{i k}\right) \leq C S T
$$

where $P_{r}\left(d_{i k}\right)$ is the power received from another concurrent transmitter $k, d_{i k}$ is the distance between stations $i$ and $k$, and int is the set of active interferers. If the received power at node $i$ is above its CST, the medium is reported busy.

The carrier sensing range $R_{s}$ can be represented as a circular block area around the transmitter following the expression

$$
R_{s}=d_{0}\left(\frac{P_{0}}{C S T}\right)^{\frac{1}{\gamma}}
$$

where $P_{0}$ is the power received at reference distance $d_{0}{ }^{2}$ and $\gamma$ is the path loss exponent. $R_{s}$ can be understood as the minimum distance allowed between two concurrent transmissions in the same channel. A large $R_{s}$ can reduce the spatial reuse and can affect the overall throughput of the network by unnecessarily abstaining nodes from transmitting due to increased sensitivity.

At a local level, if two transmitters are present within each other's range and if each of their intended receivers are within range of their transmitters only, they could be allowed to transmit simultaneously. But in contradiction, $R_{s}$ can hinder and allow only one transmission to occur.

To highlight the problem in hand, we consider a simple example as represented in Figure 1. This example includes two neighboring WLANs. Stations $A$ and $B$ (which are present in close vicinity) are concurrently transmitting to $A P 1$ and $A P 2$ respectively. By utilizing the normal PCS method, both $A$ and $B$ use a constant CST value and thus lie in each other's carrier sensing range (represented with regular circles). Link $l_{1}$ and $l_{2}$ are not allowed to operate simultaneously. However, if the carrier sensing range of $A$ and $B$ is decreased (by increasing the CST), both links could coexist to operate. The dashed circles represent the new carrier sensing range. This change in CST to accommodate for concurrent transmission is the underlying idea behind the design of DSC algorithm.

As shown later in $\mathrm{V}$, in a dense environment, exposed node problem can be more dominant than the hidden node problem. For this reason, in the following we study a dynamic CST

\footnotetext{
${ }^{2}$ All stations are assumed to be using fixed transmit power (i.e. $P_{t x}$ )
} 


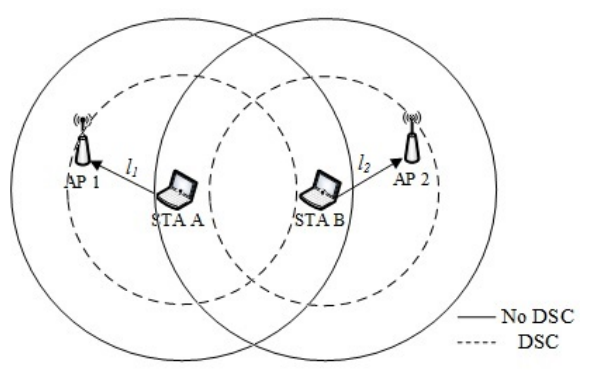

Fig. 1: Carrier sense problem of closely located stations in wifi infrastructural network.

adaptation mechanism to increase the number of concurrent transmissions (by reducing exposed nodes) as a way to improve network efficiency in dense scenarios.

\section{Dynamic Sensitivity Control Algorithm (DSC)}

As shown in section II, increase in CST results in more concurrent transmissions to take place and vice versa. Obtaining a single optimal CST for all devices in IEEE 802.11 dense networks may not be a feasible solution because nodes are not always available at the desired location if they need to make possible transmissions. Furthermore, interference levels are also variable due to random positions and also due to the variability in penetration losses. Thus, in order to optimize the system performance, there is a need of optimum carrier sensing range that should be employed at each node which can balance the spatial reuse and the impact of collisions.

In this section, we give an overview of DSC algorithm proposed in [11] for IEEE 802.11ax. Furthermore, we present a flow chart that highlights the basic operability of DSC algorithm over non-AP stations in infrastructure-based WLAN. DSC method is proposed for throughput improvements within dense implementation of relatively small cells (e.g. a dense apartment blocks). The basic idea of DSC is to vary CST levels at each station in a distributed manner (avoiding signaling overhead of centralized approaches) within the network; i.e. stations that are placed near to their respective AP can have higher CST/lower carrier sensing range because interference from concurrent transmissions would have limited implications (due to capture effect), while stations that are placed further away should have lower CST/higher carrier sensing range because the probability of correct transmissions can be increased by reducing the presence of hidden nodes.

In an infrastructure-based WLAN scenario, stations are associated to their respective AP. Each station receives beacons from their AP at every Beacon Interval (BI) seconds and accumulates the measured Received Signal Strength Indication (RSSI) of each received beacon. The Flow chart of DSC algorithm used at each non-AP station is illustrated in Figure 2. For every beacon frame received, the station accumulates the RSSI up-till the UpdatePeriod. This UpdatePeriod time is a preset value that encompasses multiple BI (i.e. if it is set to 1 second and the BI is set to $100 \mathrm{~ms}$, then 10 beacons are expected to be received). The DSC algorithm maintains a moving average of RSSI (called AvgRSSI) of all received beacons within the UpdatePeriod. If beacon frame is not received within a BI, BeaconCount (the number of consecutive beacons missed) is incremented. It is compared with BeaconCountLimit (limit to consecutive missed beacons). When BeaconCount $>$ BeaconCountLimit, the existing average RSSI is decremented by a default value (i.e. $R S S I D e c$ ), this will effectively increase the carrier sensing range and the probability of receiving beacons successfully.

After every UpdatePeriod, each station tunes its CST. In the first step, Margin is subtracted from the AvgRSSI so as to set the CST. In the next steps, the new calculated CST is confined between an upper limit (UpperLimit) and lower limit (LowerLimit) so that if the station is located near its AP, it is assigned a CST that falls near the upper limit and vice versa.

The above mentioned DSC algorithm effectively allows

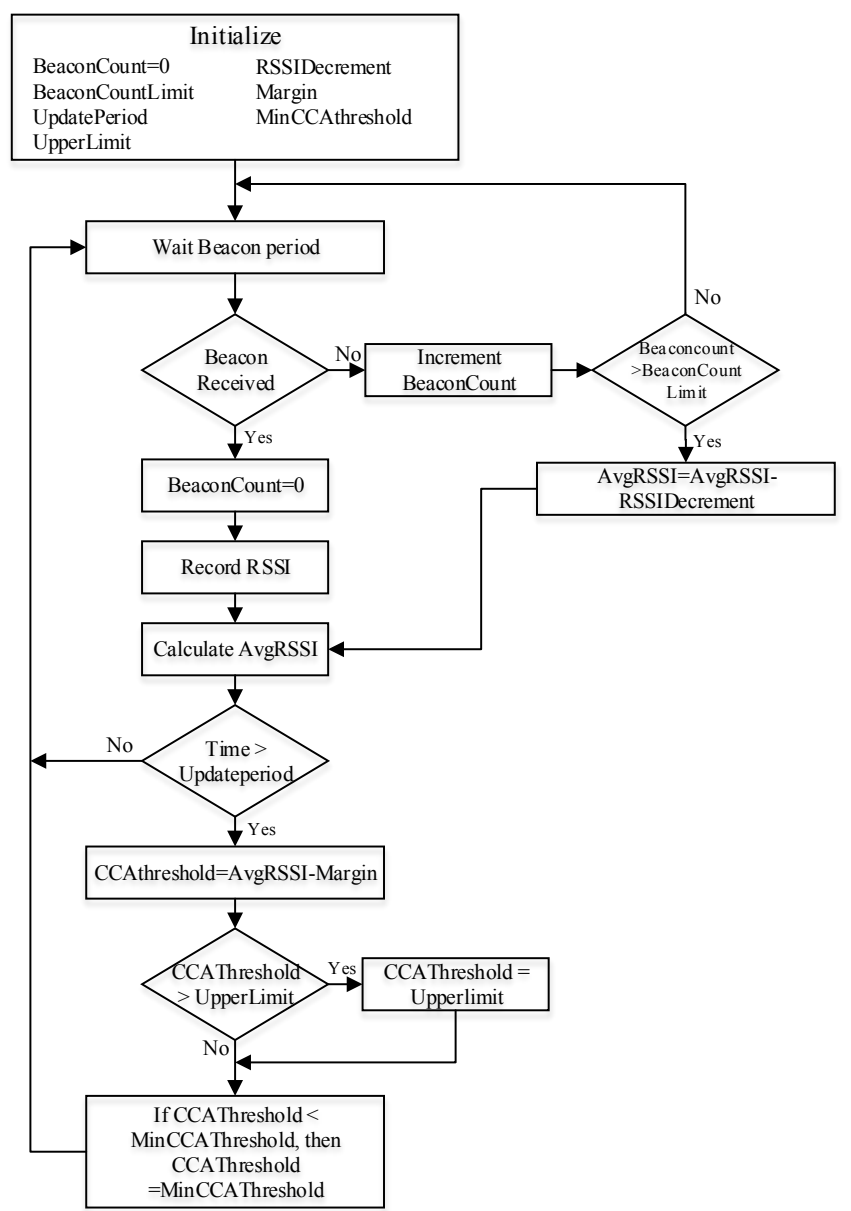

Fig. 2: Flow chart of DSC algorithm used at each non-AP station.

more flows to coexist and, as shown in section $\mathrm{V}$, this results in higher per flow and aggregate throughput.

\section{Simulation SETUP}

We present a simulation-based study to evaluate the performance of IEEE 802.11 infrastructure network operated within dense building apartment. We compare the performance when DSC was used within the network with the legacy IEEE 802.11 in which a constant CST threshold was set in every node. 
In our simulations, we considered the scenario defined by the IEEE 802.11ax WG in [15] consisting in a multifloor residential building (see Figure 3). It consisted of 100 apartments and had the following specifications:

- 5 floors, $3 \mathrm{~m}$ height of each floor

- $2 \times 10$ apartments in each floor

- Apartment size: $10 \mathrm{~m} \times 10 \mathrm{~m} \times 3 \mathrm{~m}$

- Building type: Residential

- External wall type: Concrete with windows

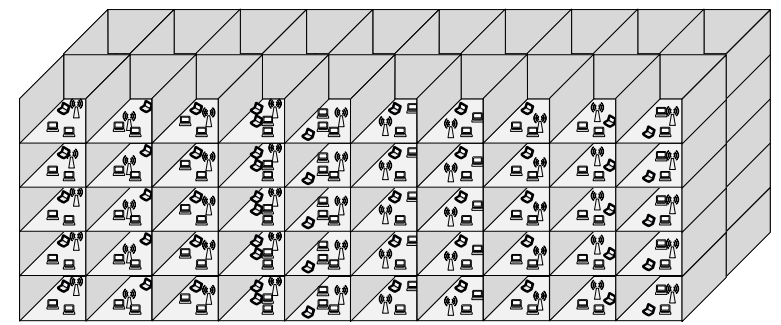

Fig. 3: Layout of dense deployment of IEEE 802.11 infrastructural network in residential building.

A single AP was randomly placed within the walls of each apartment. Five non-AP stations were placed around each AP randomly. Furthermore, APs selected channel 1, 6 and 11 randomly so that each channel was shared by $1 / 3$ of the cells. We focus our study on the use of $2.4 \mathrm{GHz}$ band because this band is more restricted in dense environments. The simulation was carried out using NS-3 network simulator in which Hybrid building propagation loss model was used [16]. For the final calculated results, a large enough number of simulations were run in order to have small $95 \%$ confidence intervals. A large enough simulation time was chosen to disregard the transient time due to initial association between stations and APs. We considered uplink transmission ${ }^{3}$, where each non-AP station was in saturation condition ${ }^{4}$ (i.e. stations always have frames to transmit). Constant Bit Rate UDP flows were used on each transmitting node. It is important to mention here that the comparison between DSC and conventional IEEE 802.11 network was done under the exact same network conditions. We modified the ns-3 simulation package, a) to allow station to measure the received energy level of each beacon frame received from the relevant $\mathrm{AP}, \mathrm{b}$ ) by improving hybrid building pathloss model to accommodate for floor penetration losses.

The metrics used in our evaluation are: 1) aggregate throughput (total bytes correctly received by the receivers per second); 2) Frame Error Rate (FER); 3) Fairness (calculated according to Jains fairness index [17]); 4) number of hidden nodes; 5) number of exposed nodes. For the hidden node analysis, we considered pair of hidden nodes (i.e. two nodes that are hidden from each other) as a single entry. This simplification was also used for the exposed node count.

The description of Physical and MAC layer parameter used within our simulation are detailed in Table I.

The details of parameters used in the DSC algorithm are highlighted in Table II.

\footnotetext{
${ }^{3}$ We evaluate DSC over uplink transmissions because it is the worst case in terms of contention.

${ }^{4}$ Saturation is used to explore maximum capacity.
}

TABLE I: Physical and MAC layer parameters for simulation.

\begin{tabular}{|c|c|c|c|}
\hline Parameter & Values & Parameter & Values \\
\hline Wireless Standard & $\begin{array}{c}\text { IEEE802.11g and } \\
\text { IEEE802.11n }\end{array}$ & Packet size & 1000 bytes \\
\hline Frequency band & $2.4 \mathrm{GHz}$ & Trasmission power & $16.0206 \mathrm{dBm}$ \\
\hline $\begin{array}{c}\text { Physical } \\
\text { transmission rate } \\
\text { for IEEE 802.11g }\end{array}$ & $24 \mathrm{Mbps}$ & Antenna gain & $1 \mathrm{~dB}$ \\
\hline $\begin{array}{c}\text { Physical } \\
\text { transmission rate } \\
\text { for IEEE 802.11n }\end{array}$ & $\begin{array}{c}\text { i. 7.2Mbps, } \\
\text { ii.28.9Mbps, } \\
\text { iii.72.2 Mbps }\end{array}$ & Noise figure & $7 \mathrm{~dB}$ \\
\hline $\begin{array}{c}\text { Propagation loss } \\
\text { model }\end{array}$ & $\begin{array}{c}\text { Hybrid buildings } \\
\text { propagation loss }\end{array}$ & $\begin{array}{c}\text { Energy detection } \\
\text { threshold }\end{array}$ & $-78 \mathrm{dBm}$ \\
\hline $\begin{array}{c}\text { Wall penetration } \\
\text { loss }\end{array}$ & $12 \mathrm{~dB}$ & $\begin{array}{c}\text { Initial CCA } \\
\text { threshold }\end{array}$ & $-80 \mathrm{dBm}$ \\
\hline $\begin{array}{c}\text { Floor penetration } \\
\text { loss }\end{array}$ & $17 \mathrm{~dB}$ & $\begin{array}{c}\text { CCA threshold for } \\
\text { APs }\end{array}$ & $-80 \mathrm{dBm}$ \\
\hline $\begin{array}{c}\text { Auto Rate Fallback } \\
\text { (ARF) }\end{array}$ & not used & $\begin{array}{c}\text { Guard interval } \\
\text { Chat }\end{array}$ & Long \\
\hline Data preamble & Short & Aggregation & not used \\
\hline Beacon Interval & $100 \mathrm{~ms}$ & & \\
\hline
\end{tabular}

TABLE II: Parameters used in DSC algorithm.

\begin{tabular}{|c|c||c|c|}
\hline Parameter & Values & Parameter & Values \\
\hline BeaconCount Limit & 5 & Updateperiod & 2 seconds \\
\hline Upperlimit & $-40 \mathrm{dBm}$ & MinCCA threshold & $-82 \mathrm{dBm}$ \\
\hline
\end{tabular}

\section{Simulation Results AND Discussion}

In this section, we report on the experimental evaluation of DSC algorithm. We compare the IEEE 802.11 network that utilizes DSC algorithm with the network that utilizes legacy IEEE 802.11 devices. In the following sections, we demonstrate that DSC algorithm provides multifold benefits in dense IEEE 802.11 implementations.

\section{A. Selection of recommended parameters for DSC algorithm}

First, we evaluate the DSC algorithm to uncover the combination of recommended values for Margin and RSSIDec which provide maximum efficiency. Different combination of values for Margin (5, 10, 15, 20 and 25) and RSSIDec (4, 5 and 6) are used for the evaluation process.

In this section, we define a network in which all non-AP stations implement DSC and utilize a fixed data rate (24Mbps). We then compare different metrics to the same network with all stations using constant CST.

Figure 4a. presents the percentage increase in aggregate throughput for all the nodes while utilizing different set of Margin and RSSIDec. The throughput results indicate around $10 \%$ improvements for all the cases over the conventional IEEE 802.11 protocol.

Figure $4 \mathrm{~b}$. shows the \% increase in fairness achieved while utilizing DSC algorithm. The proposed algorithm increases the aggregate throughput along with fairness in the system. Maximum fairness benefits are achieved when lower values of Margin are used.

Figure 4c. highlights the \% increase in hidden nodes while utilizing DSC. At higher Margin values, the increase in hidden nodes is smaller. Another important outcome is that the presence of exposed nodes is driven to 0 .

As a consequence of the increased number of hidden nodes, the overall FER in the network is also increased. These results are highlighted in Figure 4d. It is important to mention that higher values of Margin and RSSIDec result in smaller FER degradation. This is due to the fact that the impact of Margin and RSSIDec results in lower CST and thus the carrier sensing range is increased. As a consequence, the FER is decreased due to less hidden nodes. 


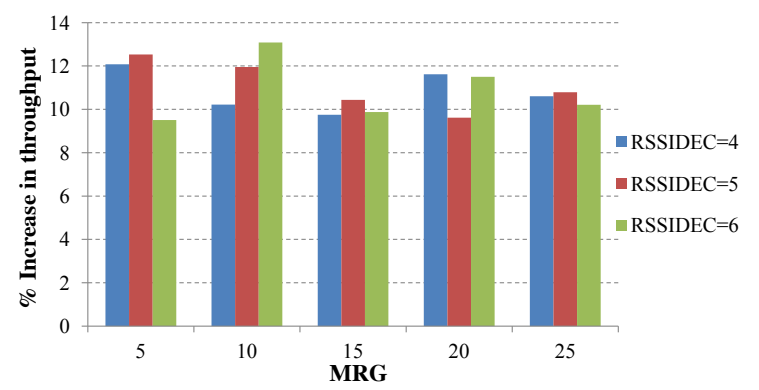

(a) Throughput improvement with DSC.

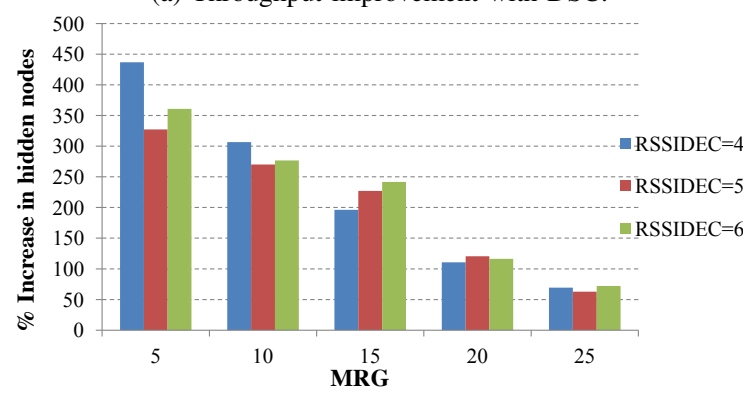

(c) Increase in number of hidden nodes with DSC

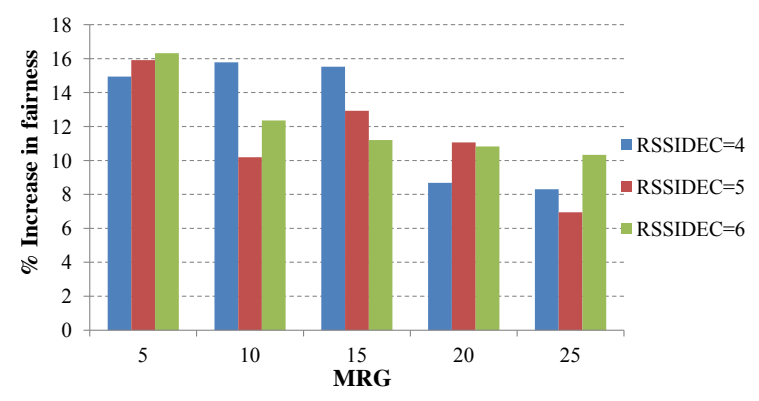

(b) Fairness improvement with DSC

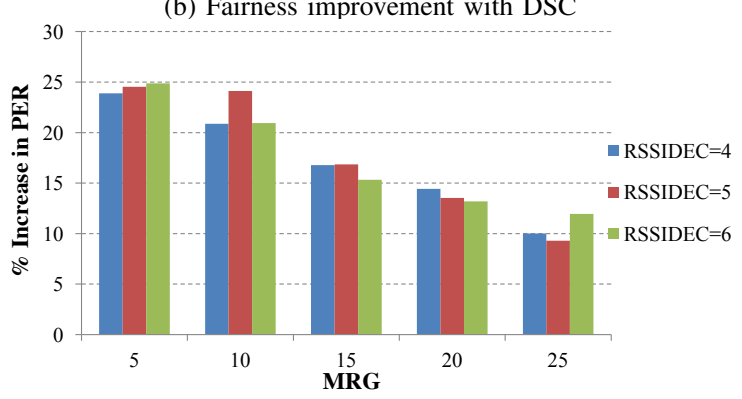

(d) Increase in FER with DSC

Fig. 4: Increase of different metrics when DSC is in use for different combinations of Margin and RSSIDec.

Comparing Figure Figure $4 a, 4 b, 4 c$ and $4 d$, it is pertinent to mention that the DSC scheme provides improvements in throughput and fairness at the cost of increasing FER and hidden nodes. After closely analyzing the results, we chose Margin as 20 and RSSIDec as 6 to be the recommended parameters that create a balance between the negative and positive aspects of DSC. We employ these values for DSC algorithm in the remainder of the paper.

\section{B. Combining DSC with Channel Selection and Rate Control}

In this section, we evaluate the performance of DSC under channel selection and rate control. As mentioned in [18], the DSC algorithm can be combined with an intelligent channel selection to provide increased efficiency. We further assess the DSC algorithm varying the Modulation and Coding Scheme (MCS) at each non-AP.

We simulate the following scenarios to expose a comparison between IEEE 802.11 network utilizing and not utilizing DSC algorithm, a) IEEE 802.11n network with Fixed MCS and Random CHannel Selection (RCHS+FMCS), b) IEEE 802.11n network with OPtimal CHannel Selection ${ }^{5}$ and Fixed MCS (OPCHS+FMCS), c) IEEE 802.11n network using Random MCS and Random CHannel Selection (RCHS+RMCS), d) IEEE 802.11n network with OPtimal CHannel Selection and Random MCS (OPCHS+RMCS).

Random MCS is added to emulate the presence of inner walls and other obstacles within the apartment/office that will trigger the rate adaptation on stations receiving varying signal quality. For optimal channel selection, three $20 \mathrm{MHz}$-wide nonoverlapping channels (i.e 1, 6, and 11) are used. For fixed MCS case, the MCS index used is 7 (i.e. PHY rate of 72.2 Mbps) and for random MCS, a random MCS index (following uniform distribution) is selected among 0,3 and 7 (i.e. 7.2, 28.9 and $72.2 \mathrm{Mbps}$ ) for all the nodes in the network.

\footnotetext{
${ }^{5}$ In optimal channel selection, channels are selected at each AP so that the distance between co-channel cells is maximized.
}

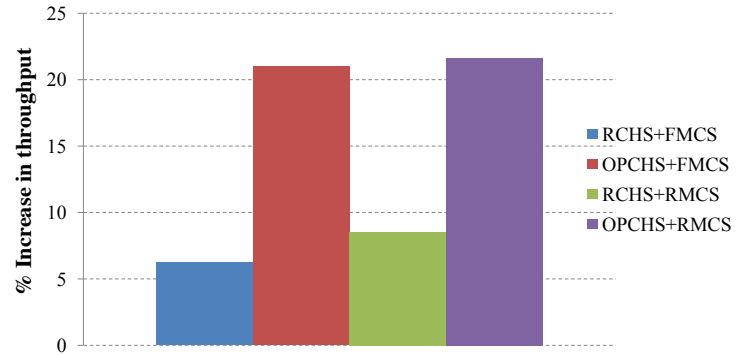

Fig. 5: Comparison of four schemes in terms of \% increase of throughput while utilizing DSC.

1) Throughput comparison: In Figure 5, we illustrate a comparison of throughput improvements induced by the use of DSC in a dense IEEE 802.11n WLAN. It is worth noting that the scenarios where DSC is combined with optimal channel selection provide maximum throughput gains of more than $20 \%$. Furthermore, note that when MCS is set randomly, the average MCS on the network is lower (i.e. transmissions last longer) and the penalty imposed by exposed nodes is higher. In those cases, DSC has more room for improvement.

2) Fairness analysis: It is logical to think that DSC may decrease fairness by giving more transmission opportunities to nodes that are near the AP, since they set higher $C S T$ values. On the other hand, DSC reduces the number of exposed nodes, which may become starved when they are located between two unsynchronized transmitters. Figure 6 indicates that fairness is increased in all the scenarios when DSC is used. This validates our previous conclusion that DSC increases the aggregate throughput by fairly increasing throughput over all the nodes.

3) FER assessment: In Figure 7 the average FER value of the four scenarios is presented, while comparing the scenarios with and without DSC. As mentioned in section V-A, FER is increased when DSC is introduced. FER is slightly improved 


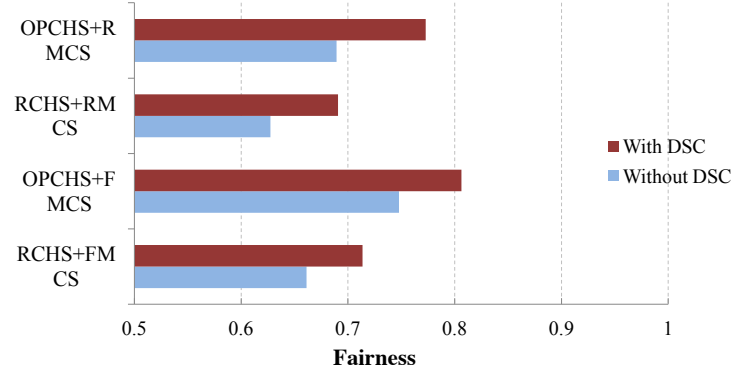

Fig. 6: Fairness comparison between four schemes w.r.t average values.

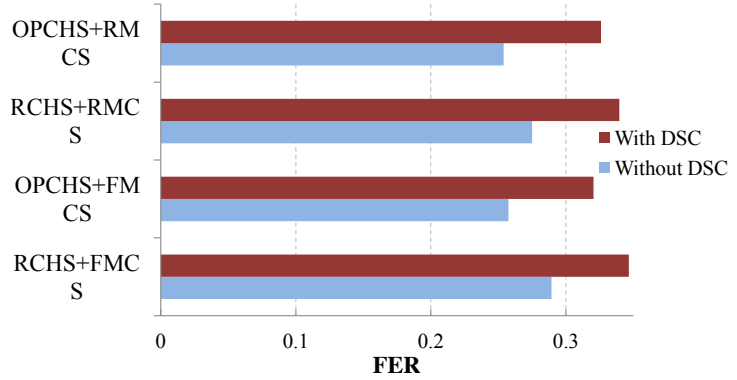

Fig. 7: FER comparison of four schemes while utilizing DSC.

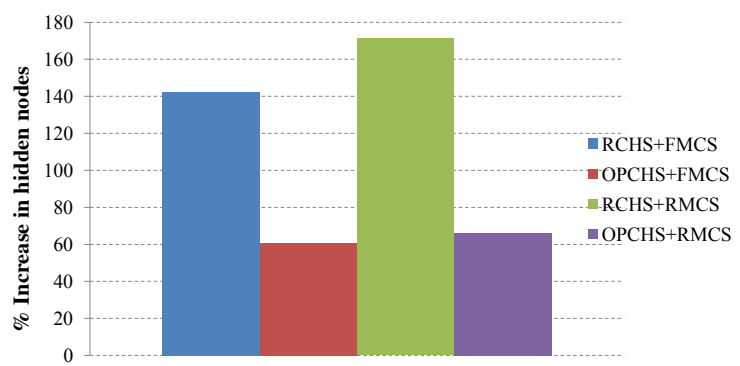

Fig. 8: Comparison of four schemes in terms of $\%$ increase in hidden nodes while utilizing DSC.

when optimal channel selection is used.

4) Hidden and exposed nodes comparison: As expected, $\%$ increase in hidden nodes is smaller while utilizing optimal channel selection. This result is depicted in Figure 8. With a random channel selection, the increase in hidden nodes is around $150 \%$. On the contrary to hidden nodes, in all of these experiments we witness almost $100 \%$ decrease in the number of exposed nodes.

\section{CONCLUSION}

In this paper, we investigated the DSC algorithm proposed for IEEE 802.11ax to improve the network efficiency in dense deployments. We described the detail functionality of DSC, an algorithm that dynamically adapts the carrier sense range. Furthermore, we used NS-3 simulator to evaluate the benefits provided by DSC, as compared to legacy IEEE 802.11. The analysis of the results was used to extract the recommended parameter tuning for the DSC algorithm. Detailed simulation results indicated that DSC allowed multiple concurrent transmissions to coexist and thus increased the overall throughput over the cost of increased hidden nodes and FER. Directions of future work include the design of DSC scheme to operate at AP and to analyze the impact of DSC in downlink transmissions. For the sake of brevity, detailed analytical evaluation of DSC scheme is not included in this paper and will be provided in our future work. Further study of DSC algorithm in different environments (other than residential), under less dense scenario and infrastructural hybrid environment (consisting of legacy and DSC stations) will be a useful step to this research.

\section{ACKNOWLEDGMENTS}

This work was partially supported by the ERDF and the Spanish Government through project TEC2012-32531.

\section{REFERENCES}

[1] I. S. Association, "IEEE standard for information technologytelecommunications and information exchange between systems local and metropolitan area networks-specific requirements part 11: Wireless lan medium access control (mac) and physical layer (phy) specifications," ANSI/IEEE Std. 802.11-2012, 2012.

[2] C. Thorpe and L. Murphy, "A survey of adaptive carrier sensing mechanisms for ieee 802.11 wireless networks," Communications Surveys Tutorials, IEEE, vol. 16, no. 3, pp. 1266-1293, Third 2014.

[3] I. Jamil, L. Cariou, and J.-F. Helard, "Improving the capacity of future ieee 802.11 high efficiency wlans," in Telecommunications (ICT), 2014 21st International Conference on, May 2014, pp. 303-307.

[4] R. Madan, A. Sampath, and N. Khude, "Enhancing 802.11 carrier sense for high throughput and qos in dense user settings," in Personal Indoor and Mobile Radio Communications (PIMRC), 2012 IEEE 23rd International Symposium on, Sept 2012, pp. 253-259.

[5] S. Merlin and S. Abraham, "Methods for improving medium reuse in ieee 802.11 networks," in Consumer Communications and Networking Conference, 2009. CCNC 2009. 6th IEEE, Jan 2009, pp. 1-5.

[6] O. Acholem and B. Harvey, "Throughput performance in multihop networks using adaptive carrier sensing threshold," in IEEE SoutheastCon 2010 (SoutheastCon), Proceedings of the, March 2010, pp. 287-291.

[7] Y. Zhang, C. Assi, B. Alawieh, and H. Alazemi, "A spatiotemporal contention resolution for enhancing spatial reuse in wireless networks," Vehicular Technology, IEEE Transactions on, vol. 60, no. 2, pp. 680691, Feb 2011.

[8] C. Thorpe, S. Murphy, and L. Murphy, "Ieee802.11k enabled adaptive carrier sense management mechanism (kapcs2)," in Integrated Network Management (IM), 2011 IFIP/IEEE International Symposium on, May 2011, pp. 509-515.

[9] Status of ieee 802.11 hew study group high efficiency wlan (hew). [Online]. Available: http://www.ieee802.org/11/Reports/hewupdate.htm

[10] "802.11 hew sg proposed par," IEEE 802.11ax, IEEE 802.11-14/0165ro.

[11] G. Smith, "Dynamic sensitivity control-v2," IEEE 802.11ax, IEEE 802.11-13/1012r4.

[12] The network simulator- ns-3. [Online]. Available: www.nsnam.org/

[13] L. B. Jiang and S.-C. Liew, "Improving throughput and fairness by reducing exposed and hidden nodes in 802.11 networks," Mobile Computing, IEEE Transactions on, vol. 7, no. 1, pp. 34-49, Jan 2008.

[14] A. Kochut, A. Vasan, A. Shankar, and A. Agrawala, "Sniffing out the correct physical layer capture model in 802.11 b," in Network Protocols, 2004. ICNP 2004. Proceedings of the 12th IEEE International Conference on, Oct 2004, pp. 252-261.

[15] J. R., "IEEE 802.11 tgax simulation scenarios," IEEE 802.11ax, IEEE 802.11-14/0621r3.

[16] Hybrid buildings propagation loss model: ns3-design document. [Online]. Available: http://www.nsnam.org/docs/models/html/buildingsdesign.html

[17] J. R., "Fairness: How to measure quantitatively?" ATM Forum/94-0881, Sept. 1994.

[18] G. Smith, "Dense apartment complex capacity improvements with channel selection and dynamic sensitivity control," IEEE 802.11ax, IEEE 802.11-13/1487r2. 